\title{
Streptococcal Toxic Shock Syndrome Presenting as an Acute Abdomen
}

\author{
Christy Anthony, ${ }^{1,2}$ Jeffrey M. Tessier, ${ }^{3}$ James Sanders, ${ }^{4}$ Daniel Ziegler, ${ }^{1}$ and Therese M. Duane ${ }^{1}$
}

\begin{abstract}
Background: Streptococcal toxic shock syndrome is a potentially fatal complication of group A streptococcal (GAS; Streptococcus pyogenes) infection, but is infrequently reported with primary peritonitis caused by this pathogen. Case: A 72-y-old white male developed acute abdominal pain accompanied by septic shock, acute kidney injury, and coagulopathy. Surgical examination revealed peritonitis and blood cultures grew GAS. His clinical deterioration did not respond to antibacterial therapy; repeat laparotomy discovered extensive dead bowel and he died from his illness.

Conclusion: Toxic shock syndrome may cause septic shock in patients with primary peritonitis caused by GAS. The coagulopathy ascribed to this syndrome may lead to bowel ischemia. Clinicians should be aware of the potential complications associated with invasive GAS infections.
\end{abstract}

G ROUP A STREPTOCOCCAL (GAS) infections cause substantial morbidity and mortality among human populations around the world. Streptococcal toxic shock syndrome (STSS) is one of the deadliest forms of GAS infection, primarily because of delayed diagnosis and a lack of highly effective therapeutic interventions, with a mortality of $40 \%-$ $80 \%$ [1]. The U.S. Centers for Disease Control and Prevention (CDC) 2010 case definition of STSS includes isolation of GAS in culture, hypotension, and evidence for multi-organ dysfunction (kidney, liver, or lung injury; coagulopathy; soft tissue necrosis; or a generalized erythematous macular skin rash) [1]. The most common forms of GAS infection associated with STSS are skin/soft tissue infections (SSTIs) [1]. We describe a fatal case of STSS arising from primary peritonitis, an unusual presentation for a rare but aggressive disease.

\section{Case Review}

A 72-year-old healthy male developed worsening abdominal pain six hours prior to hospital presentation. He reported some physical exercise in the hours preceding the onset of the pain and denied any nausea, vomiting, or change in bowel habits. His past history included mild dementia with depression. At presentation, he was found to be hypotensive (blood pressure $90 / 40 \mathrm{~mm} \mathrm{Hg}$ ) and tachycardic. His examination was significant for signs of peritonitis, as well as an isolated patch of blanchable erythema and induration of his right medial thigh. Examination of his scrotum revealed ecchymoses and erythema along the dependent portion with non-tender and appropriately positioned testicles. Laboratory tests were significant for a white cell count of 1,520 per microliter, platelets 103,000 per microliter, serum creatinine of $4.8 \mathrm{mg} / \mathrm{dL}$, lactic acid of $8.4 \mathrm{mmol} / \mathrm{L}$, an of 1.72 , and a procalcitonin of $46.07 \mathrm{ng} /$ $\mathrm{mL}$. Computed tomography (CT) imaging of the abdomen/ pelvis revealed significant stranding in the right lower quadrant down into the pelvis, right perinephric area, right inguinal area, scrotum, and right thigh (Fig. 1). He received aggressive fluid resuscitation, pressor support, intravenous vancomycin, and piperacillin-tazobactam. He was taken emergently to the operating room because of his worsening clinical status, septic shock, and acute abdomen.

The initial diagnostic laparoscopy revealed diffuse intraperitoneal purulence, so an exploratory laparotomy was performed, the purulent material was evacuated, and the

\footnotetext{
${ }^{1}$ Department of General Surgery and Surgical Critical Care, ${ }^{3}$ Antimicrobial Stewardship Program, ${ }^{4}$ Department of Pharmacy, John Peter Smith Hospital, Fort Worth, Texas.

${ }^{2}$ Department of Surgery, Baylor University Medical Center, Dallas, Texas.

(C) Christy Anthony et al. 2016; Published by Mary Ann Liebert, Inc. This Open Access article is distributed under the terms of the Creative Commons License (http://creativecommons.org/licenses/by/4.0), which permits unrestricted use, distribution, and reproduction in any medium, provided the original work is properly credited.
} 


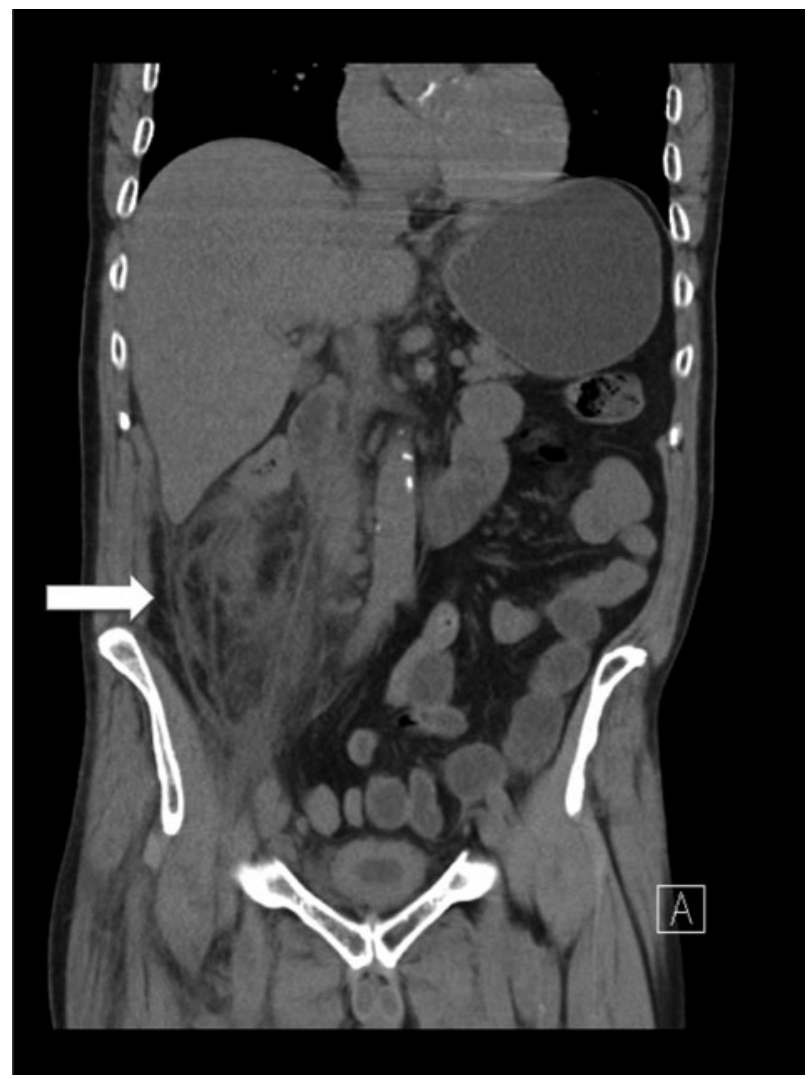

FIG. 1. Computed tomography coronal view of the abdomen. The white arrow highlights stranding in the right lower quadrant, extending along the right paracolic gutter into the pelvis.

bowel was inspected. There were no ulcerations, bowel perforations, or ischemic/necrotic areas along the small bowel and colon, but there were thromboses of the small venules of the omentum. Given the CT findings, the right retroperitoneum was explored but no obvious pathology was identified. Peri-operatively he remained hemodynamically unstable and laboratory testing revealed lactic acidosis with an arterial blood pH of 7.19, and an increased INR of 2.75. Post-operatively, the patient continued to require pressor support and end organ damage was evident on laboratory findings. A Gram stain of his initial blood culture revealed gram positive cocci in pairs, causing suspicion for STSS, as a result, penicillin $\mathrm{G}$ and clindamycin were administered. Because of his worsening lactic acidosis, the patient was again taken to the operating room and was found to have extensive, foul-smelling ischemic small bowel and necrosis of the entire colon. Comfort care was initiated after discussion of these findings with his family and he died soon after cessation of aggressive care. The intra-operative peritoneal fluid cultures were negative, but his initial two sets of blood cultures grew GAS.

\section{Discussion}

Our report describes a patient who presented with an illness characterized by rapid onset, relentless clinical deterioration despite aggressive medical and surgical interventions, and meeting the CDC 2010 case definition for STSS, including the isolation of GAS from blood cultures, hypotension, and the development of multi-organ dysfunction (acute kidney injury, coagulopathy). Our patient had primary peritonitis and, importantly, had early evidence of a coagulopathy on presentation, with an elevated INR and the presence of multiple thrombosed venules in the omentum at the time of his first exploratory laparotomy. Unfortunately, these venular thromboses were likely harbingers of the subsequent vascular insult leading to extensive bowel ischemia, worsening lactic acidosis, and death. Our patient reported weightlifting in the hours preceding the onset of his abdominal pain. This information may be relevant to the pathogenesis of his infection, because damaged skeletal muscle has been shown to increase vimentin expression, leading to increased binding of GAS organisms, a process enhanced by non-steroidal anti-inflammatory drugs [2].

Primary peritonitis is infrequently caused by GAS and has rarely been associated with toxic shock syndrome. Westwood and Roberts [3] performed a systematic review of all published adult cases $(n=32$ patients) of primary GAS peritonitis, covering the period from 1990 to 2011. The mean age was $38 \mathrm{y}$, with a male:female ratio of $1: 4$, with an unidentified primary source of infection in one-half (19/38) of the patients; seven patients had evidence for retroperitoneal inflammation on CT imaging or at the time of laparotomy, including psoas thickening, edema, and micro-abscess formation. Thirteen of the 32 patients had STSS and all 32 of these patients survived, a finding that the authors acknowledge may be related to publication bias.

Munrós et al. [4] published a recent systematic review of primary GAS peritonitis, including cases published between 1975 and 2014 ( $n=37$ patients). The mean age in this series was $32 \mathrm{y}$ and demonstrated the same male:female ratio (1:4) as the Westwood/Roberts review [3]. The authors postulate the female predominance is because of ascending infection of the peritoneum via GAS vaginal colonization, based mainly on the finding of frequent positive genital (vaginal, cervical, endometrial) cultures from the female patients. The authors advocate laparoscopy with washout in cases of primary peritonitis, both to obtain diagnostic samples and to reduce the bacterial burden associated with these infections.

The pathogenesis of STSS has been reviewed extensively elsewhere [1], but recent studies have shed more light on bacterial factors associated with development of this syndrome. Mutations in several negative regulators of GAS virulence factors, including $\operatorname{csr} S / \mathrm{csrR}$ and $\mathrm{rgg}$ genes, have been found at a much higher frequency in STSS-associated clinical isolates $(57.3 \%)$ compared with non-invasive clinical isolates $(1.7 \%)$ [5]. These mutations are associated with increased expression of GAS virulence genes in vitro and enhanced lethality in a murine intra-peritoneal infection model in vivo [5]. Recently, Kaneko et al. [6] published a case report of primary GAS peritonitis in a 28 -y-old female caused by a GAS strain with a mutation in the $c s r S$ gene. The patient developed acute abdominal pain and diarrhea followed rapidly by septic shock, but she ultimately responded to treatment with penicillin $\mathrm{G}$ and clindamycin. Although her case did not meet the 2010 CDC definition for STSS, the fulminant course of her infection in a previously healthy young patient prompted further investigation and identification of the $c s r S$ gene mutation.

The definitive treatment of primary GAS peritonitis has not been confirmed prospectively in clinical trials. The cumulative experience described in the literature identifies several themes: (1) aggressive fluid resuscitation in patients 
exhibiting sepsis; (2) CT imaging to exclude causes of secondary peritonitis and outline the location of fluid collections; (3) laparoscopy or laparotomy to obtain diagnostic specimens and remove purulent material mechanically; and (4) initial broad spectrum antibacterial therapy aimed at the most common pathogens associated with intra-abdominal infections. When STSS is suspected or confirmed, there are no therapeutic interventions that have been identified as providing a clear survival advantage. The addition of antibacterial agents that inhibit protein toxin synthesis (e.g., clindamycin, linezolid) is advocated and has excellent biologic plausibility [1]. The administration of polyclonal intravenous immune globulin (IVIG) may have a beneficial role in toxic shock syndrome. A randomized placebo-controlled trial of high-dose IVIG $(1 \mathrm{~g} / \mathrm{kg}$ body weight day $1,0.5 \mathrm{mg} / \mathrm{kg}$ days 2 and 3 ) was terminated early because of slow accrual, but analysis of the 21 enrolled patients (10 IVIG, 11 placebo) demonstrated a higher 28-d mortality among placebo recipients (10\% versus $36 \%$ ), along with lower organ failure scores with IVIG [7]. The benefits of IVIG in STSS are biologically plausible, most obviously via immunoglobulin-mediated neutralization of the superantigens responsible for widespread T-cell activation. A recent retrospective, propensity-matched cohort study of patients with toxic shock syndrome caused by to necrotizing fasciitis (TSS-NF) was presented in oral form by Bonne at the 36th Surgical Infection Society Annual Meeting (Palm Beach, Florida, May 20, 2016); this study did not find a mortality benefit with IVIG use ( $n=164$ IVIG recipients) but did note a higher cost of hospitalization in the IVIG cohort (approximately $\$ 49,000$ additional cost). Our patient died before IVIG administration and it remains unclear if specific sub-groups of patients with TSS benefit from the use of this expensive adjunctive therapy.

\section{Conclusion}

Clinicians caring for patients with acute abdominal syndromes should maintain a high index of suspicion for GAS infection when the presentation suggests primary peritonitis, especially in patients with septic shock or those meeting the 2010 CDC criteria for STSS. Prompt initiation of broad spectrum antibacterials, including protein toxin synthesis inhibitors, and additional interventions such as surgical debridement, aggressive fluid resuscitation, and high-dose IVIG should all be pursued while awaiting microbiologic confirmation of GAS infection. Novel therapies for STSS are desperately needed for this highly fatal syndrome and will likely have to be developed without reliance upon randomized controlled trials given the scarcity of the disease and the rapid clinical course.

\section{Author Disclosure Statement}

No competing financial interests exist.

\section{References}

1. Low DE. Toxic shock syndrome: Major advances in pathogenesis, but not treatment. Crit Care Clin 2013;29:651-75.

2. Hamilton SM, Bayer CR, Stevens DL, et al. Muscle injury, vimentin expression, and non-steroidal anti-inflammatory drugs predispose to cryptic group A streptococcal necrotizing infection. J Infect Dis 2008;198:1692-1698.

3. Westwood DA, Roberts RH. Management of primary group A streptococcal peritonitis: A systematic review. Surg Infect 2013; 14:171-176.

4. Munrós J, Alonso I, Del Pino M, et al. Peritonitis primaria por Streptococcus pyogenes. Rev Esp Quimioter 2014;27: 273-278.

5. Ikebe T, Ato M, Matsumura T, et al. Highly frequent mutations in negative regulators of multiple virulence genes in group A streptococcal toxic shock syndrome isolates. PloS Pathog 2010;6:e1000832.

6. Kaneko M, Maruta M, Shikata H, et al. Acute abdomen due to group A streptococcus bacteremia caused by an isolate with a mutation in the $c s r S$ gene. J Infect Chemother 2015; 21:816-819.

7. Darenberg J, Ihendyane N, Sjölin J, et al. Intravenous immunoglobulin $G$ therapy in streptococcal toxic shock syndrome: A European randomized, double-blind, placebocontrolled trial. Clin Infect Dis 2003;37:333-340.

Address correspondence to: Dr. Jeffrey M. Tessier Department of General Surgery 1500 South Main Street Fort Worth, TX 76104

E-mail: jtessier@jpshealth.org

$\begin{aligned} & \text { Abbreviations Used } \\ & \text { CDC }=\text { U.S. Centers for Disease Control and Prevention } \\ & \mathrm{CT}=\text { computed tomography } \\ & \mathrm{GAS}=\text { group A streptococcal infection } \\ & \mathrm{INR}=\text { international normalized ratio } \\ & \mathrm{IVIG}=\text { intravenous immune globulin } \\ & \mathrm{SSTIS}=\text { skin/soft tissue infections } \\ & \mathrm{STSS}=\text { streptococcal toxic shock syndrome } \\ & \mathrm{TSS}-\mathrm{NF}=\text { toxic shock syndrome caused by to necrotizing } \\ & \text { fasciitis }\end{aligned}$

Cite this article as: Anthony C, Tessier JM, Sanders J, Ziegler D, Duane TM (2016) Streptococcal toxic shock syndrome presenting as an acute abdomen. Surgical Infections Case Reports 1:1, 82-84, DOI: 10.1089/ crsi.2016.0019 